\title{
A Collaborative Molecular Modeling Environment Using a Virtual Tunneling Service
}

\author{
Jun Lee, Jee-In Kim, and Lin-Woo Kang \\ Department of Advanced Technology Fusion, Konkuk University, Seoul 143-701, Republic of Korea \\ Correspondence should be addressed to Jee-In Kim, jnkm@konkuk.ac.kr \\ Received 17 May 2012; Accepted 8 July 2012 \\ Academic Editor: Tai Hoon Kim
}

Copyright () 2012 Jun Lee et al. This is an open access article distributed under the Creative Commons Attribution License, which permits unrestricted use, distribution, and reproduction in any medium, provided the original work is properly cited.

Collaborative researches of three-dimensional molecular modeling can be limited by different time zones and locations. A networked virtual environment can be utilized to overcome the problem caused by the temporal and spatial differences. However, traditional approaches did not sufficiently consider integration of different computing environments, which were characterized by types of applications, roles of users, and so on. We propose a collaborative molecular modeling environment to integrate different molecule modeling systems using a virtual tunneling service. We integrated Co-Coot, which is a collaborative crystallographic object-oriented toolkit, with VRMMS, which is a virtual reality molecular modeling system, through a collaborative tunneling system. The proposed system showed reliable quantitative and qualitative results through pilot experiments.

\section{Introduction}

A networked virtual environment (NVE) is a computersupported collaborative work (CSCW) environment where multiple participants can interact with each other through computer networks for enhancing performances of their collaborations. Since, NVE can overcome limit of time and space differences during face-to-face collaborations, NVE has been widely researched for collaborative computeraided design $[1,2]$. In a collaborative three-dimensional (3D) computer-aided design (CAD) system, distributed participants can work together in order for creating or modifying a 3D model in a virtual environment. A 3D biomolecular modeling system is one of promising collaborative applications, and it has also been popularly researched and developed by various research groups.

In order to design new materials and new drugs, we need to understand functions of proteins through analysis of a 3D protein structure at atomic resolution. First, it is generally determined by X-ray crystallography or NMR. Second, we can simulate the behaviors of the $3 \mathrm{D}$ molecules with equations of quantum and physics through computer simulations. Third, the 3D model of an enzyme, which is a candidate material or drug, can be used to design a higher binding affinity inhibitor against a target enzyme. Last, we can simulate the designed enzyme to have better characteristics such as higher activity and stability for industrial purpose $[3,4]$.

Since molecular modeling is a large and complicated process, its participants generally collaborate together according to their roles and familiar applications. If all participants use a same molecular system, it would work well. However, it is not a case in a real world. The participating biologists generally use their own favorite molecular modeling systems among many available systems. Therefore, real-time collaborations of the participating biologists using NVE systems are not smoothly realized in general.

In this paper, we propose a collaborative experiment environment with different molecular modeling systems. The environment consists of two collaborative systems, VRMMS (virtual reality molecular modeling system) [5-7] and Co-Coot [8]. Since the biologists can use their favorite systems with their designated roles, the proposed environment provides with a tunneling service for integrating the different collaborative applications. The proposed environment can reduce extra expenses for collaborations among multiple biologists. 


\section{Related Works}

There have been several researches on collaborative molecular modeling systems for studying and analyzing 3D biomolecular structures. BioCore [9] is a real-time collaboration tool for biomolecular modeling. It performs molecular energy simulations, real-time monitoring, communication among participants, and document management. BioCore can also combined with existing software tools such as VMD [10] (a visualization tool) and NAMD [11] (a computational system). However, BioCore strictly requires a high performance PC for effective and efficient exercises of collaborations, and the network system of BioCore is not an open standard. As a result, most researchers cannot easily combine BioCore with other applications.

In our previous papers, VRMMS was suggested to visualize 3D biomolecular structures and calculate simulation of energy minimization [5]. VRMMS was also coined a NVE system to provide various collaborative device environments such as a CAVE and a laptop [6, 7]. However, it still restricts a connection from the other open-source software.

Coot is open-source software and popularly used. It is specialized to edit 3D biomolecular structures for finding a best enzyme model in a crystallography process [12]. Using Coot as a platform, we developed a novel real-time collaboration tool, Co-Coot, to study $3 \mathrm{D}$ biomolecular structures [8]. Co-Coot can represent 3D structure models simultaneously at multiple displays in remote places, and enable multiple users to manipulate the models and conveniently communicate with other users with Co-Coot. Even though, Co-Coot is a good example of research approach to combine a collaboration module with existing open source software, it can restrict possible participations from other applications with different roles.

\section{System Overview}

We designed a system architecture of the collaborative environment as a client/server network topology. It is easy to manage collaborative communications among clients and a server. The proposed environment can be illustrated as shown in Figure 1. User 1 can enter the collaborative server and register his/her collaborative project. After the registration, other remote users can join the created project using Co-Coot or VRMMS. The participated clients can create various collaborative messages such as manipulation, rendering, editing, and ownership. These messages are capsuled in the collaborative tunnels, and sent to the server. If the collaborative server receives the messages from user 1, it directly distributes them to user 2 and also translates the Co-Coot format messages into the VRMMS format messages for user 3 and user 4. The proposed agents of Co-Coot and VRMMS provide open APIs for the collaborative tunneling services [13].

The participating researchers can perform their collaboration simultaneously to share their intermediate experimental results, discuss a future direction, or solve their difficult problems via the proposed networked virtual environment. The proposed environment offers a pessimistic concurrency
TABLE 1: Supporintg functions in VRMMS.

\begin{tabular}{ll}
\hline Functions & Detailed operations \\
\hline Manipulation & Translation, rotation, and scaling \\
Rendering & Surface, wire frame, and ball and stick \\
Simulation & Energy minimization \\
Ownership & Pessimistic concurrency control \\
File transferring & File sharing \\
Chatting & Exchange text messages \\
\hline
\end{tabular}

TABle 2: Supporintg functions in Co-Coot.

\begin{tabular}{ll}
\hline Functions & Detailed operations \\
\hline Manipulation & Translation, rotation, and scaling \\
Rendering & Wire frame \\
Editing & Add, delete, and rotate an amino acid with bonds \\
Ownership & Pessimistic concurrency control \\
File transferring & File sharing \\
Chatting & Exchange text messages \\
\hline
\end{tabular}

control mechanism, which allows accesses and manipulates shared 3D molecular models with permission from the collaborative server. During the collaborative works, any user can request the collaborative server for his/her authority over a shared molecular model. The server may allow the current request for an authority if it is available. If other user already owns the shared molecular model, the server denies the request from the user. This mechanism could avoid conflicts among multiple requests from the participating researchers.

The proposed networked virtual environment also provides a private workspace if the denied user still wants to manipulate the shared molecular model privately. With the private workspace, the user can manipulate every features of the biomolecular model and the intermediate result can be stored in a file after using the private workspace.

\subsection{Collaborative Tunneling Service. See Figure 2.}

3.2. Transformation Strategies. In order to provide transformations between two different applications, we need to define a mapping mechanism between the applications. The proposed system provides transformation strategies for the mapping mechanism. We analyzed both VRMMS and CoCoot in order to extract important functions in a collaborative 3D molecular modeling process. We modeled six functions of VRMMS and Co-Coot as described in Tables 1 and 2, respectively. Table 1 shows the selected important functions of VRMMS and Table 2 shows the extracted important functions of Co-Coot. We also described the mapping functions of these two applications in Table 3. The "Manipulation" functions of VRMMS and Co-Coot are semantically "Equal". It follows that our system treats the functions as same functions. The "ownership" function, the "file transferring" function, and the "chatting" function can be "equally" treated between VRMMS and Co-Coot. 


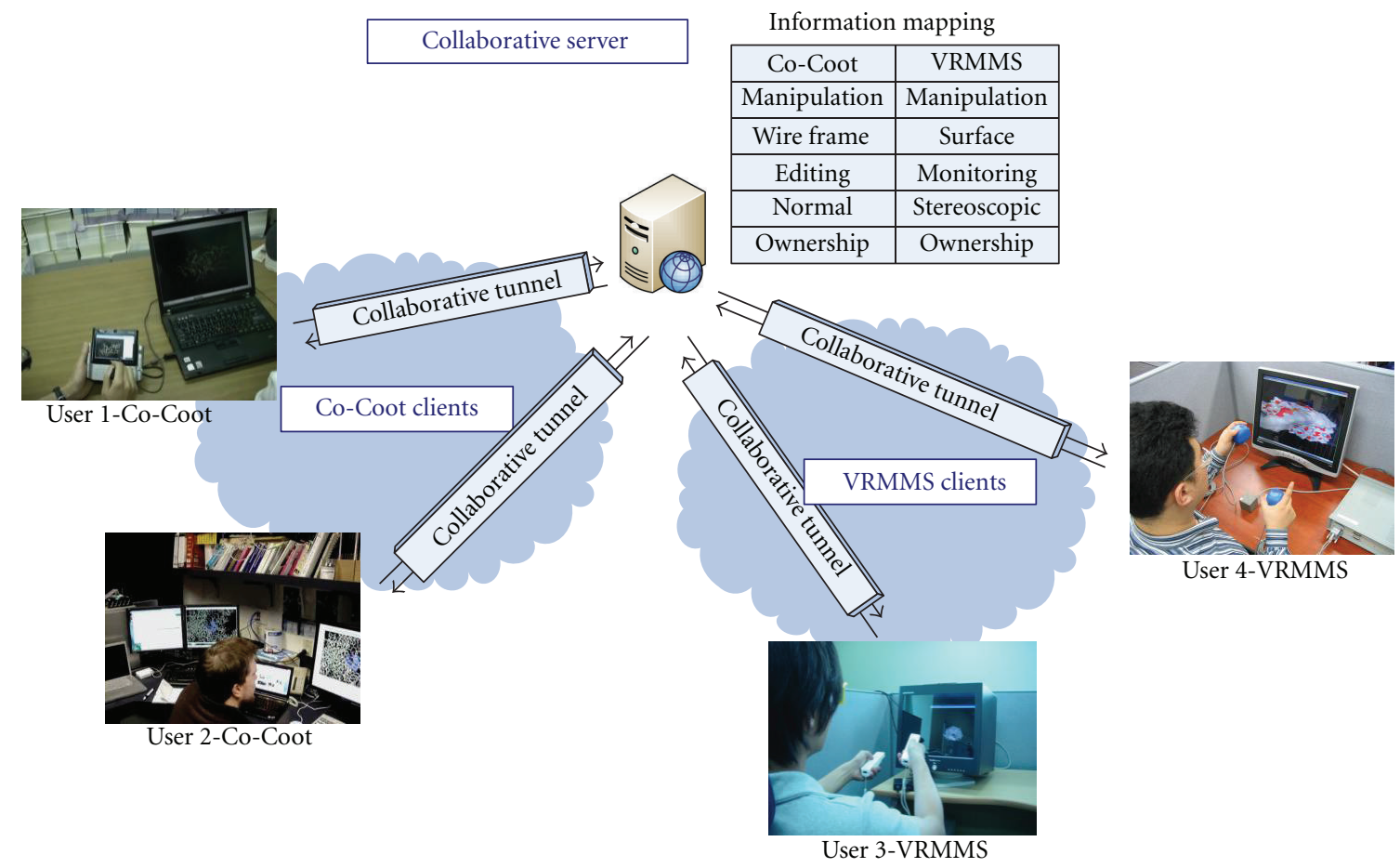

Figure 1: System overview.

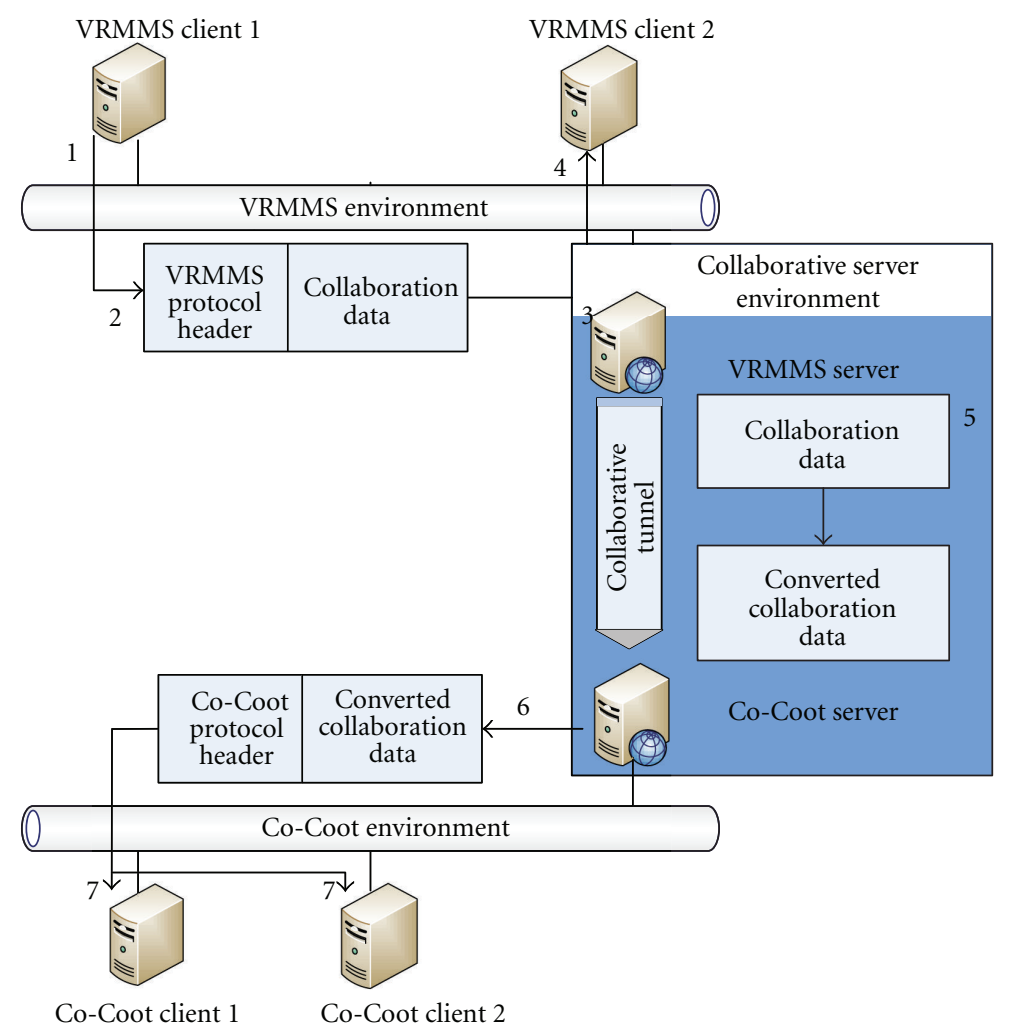

FIgURE 2: Collaborative tunneling service. 


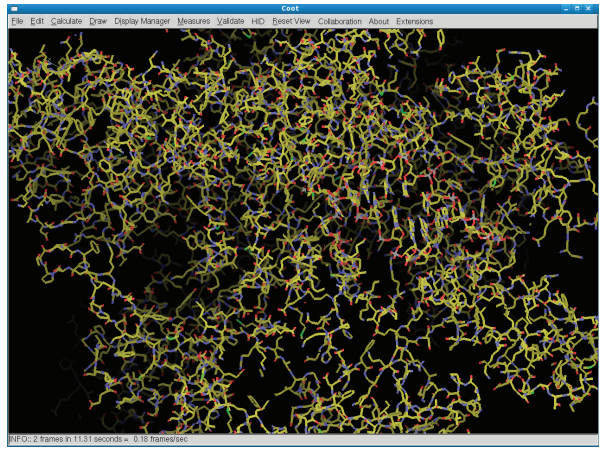

(a)

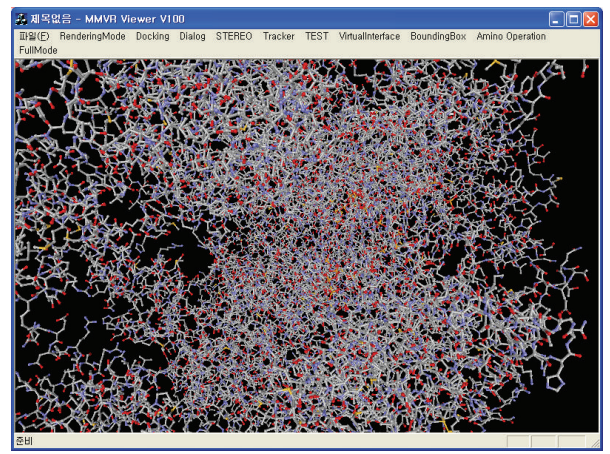

(c)

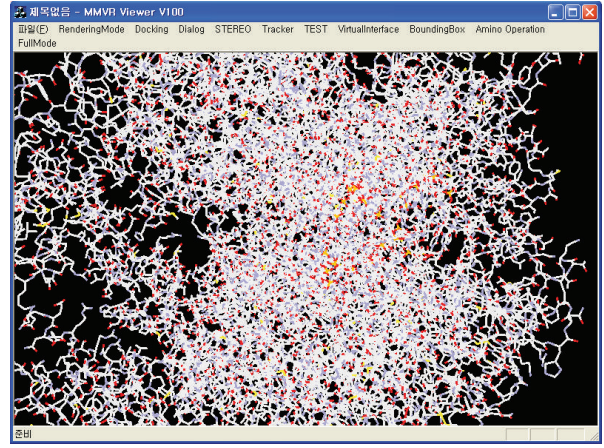

(b)

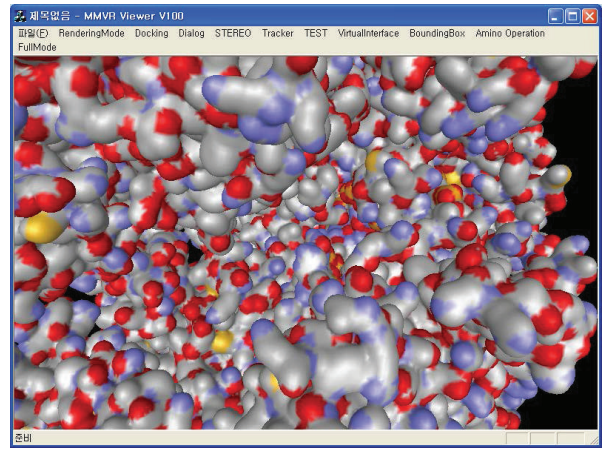

(d)

FIGURE 3: Results of visualization of 1SFO [14] (a) in the wire frame mode in Co-Coot, (b) in the wire frame mode in VRMMS, (c) in the ball and stick mode in VRMMS, and (d) in the surface mode in VRMMS.

TABLE 3: Function mapping between VRMMS and Co-Coot.

\begin{tabular}{lcc}
\hline VRMMS functions & Co-Coot functions & Semantic mapping \\
\hline Manipulation & Manipulation & Equal \\
Rendering & Rendering & Convertible \\
Simulation & - & Readable \\
- & Editing & Readable \\
Ownership & Ownership & Equal \\
File transferring & File Transferring & Equal \\
Chatting & Chatting & Equal \\
\hline
\end{tabular}

A user of VRMMS can monitor 3D molecular models with various visualization methods such as wire frame, ball and stick, and surface modes in rendering function. VRMMS also provides a simulation function to calculate energy values of the 3D molecular models. In Co-Coot, a user can visualize molecular models as a wire frame mode. Co-Coot provides an editing function to refine current 3D molecular models. Figure 3 shows different results of visualizations from the same molecular model 1SFO [14].

The "rendering" functions of the two applications are semantically and operationally different. But they are "convertible". So, a "surface" rendering model in VRMMS can be converted and expressed as a "wireframe" model in Co-Coot. The same conversion strategy can be applied to the "ball and stick" rendering model of VRMMS.
TABLE 4: Selected molecular models.

\begin{tabular}{lcc}
\hline PDB CODE & Name & Number of atoms \\
\hline 1 SFO & Yeast polymerase II & 28,649 \\
\hline
\end{tabular}

Though VRMMS has the "simulation" function, CoCoot does not, on the other hand. Then, the simulation results from VRMMS could be translated into a text format and read by the Co-Coot users as shown in Figure 4.

\section{Results of the Collaborative Experiment}

We evaluated performance of the proposed environment by several quantitative and qualitative measures. As described in Table 1, the proposed environment conducted through using a real molecular model [14]. Table 4 shows its related information such as its PDB code, name, and number of atoms.

4.1. Rendering Speed Test. First, we compared the rendering speed of Co-Coot and VRMMS with various visualizations using the same molecular models. The experiment was conducted on a desktop PC with Core 2 Quad CPU and an nVdia GTX 265 graphic card. We tested rendering speeds of Co-Coot and VRMMS for 100 seconds in terms of the FPS (frames per second) values with four possible visualization modes. As shown in Figure 5, the results showed a feasible 


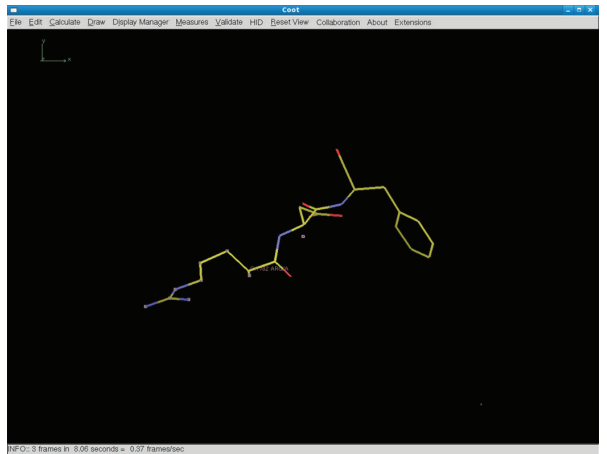

(a)

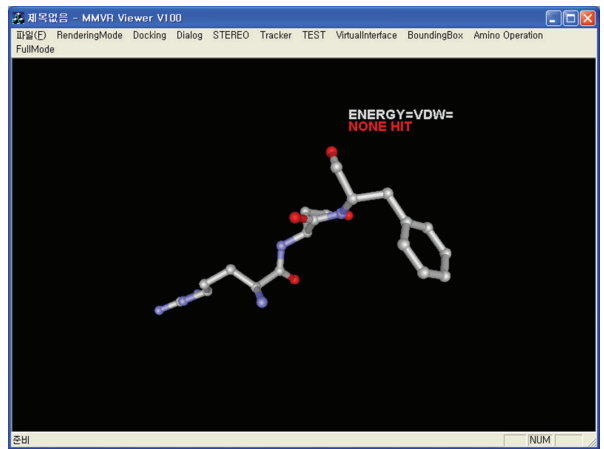

(c)

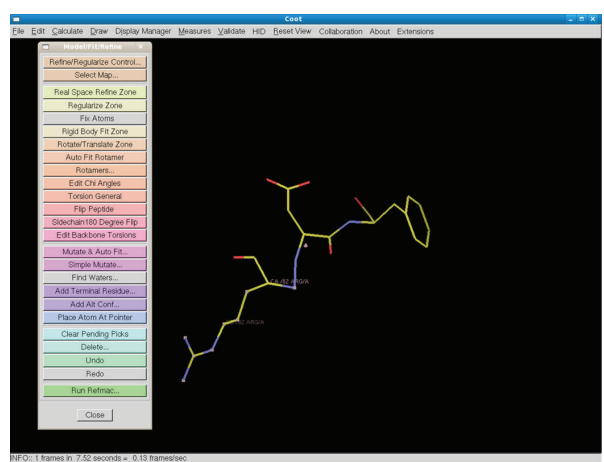

(b)

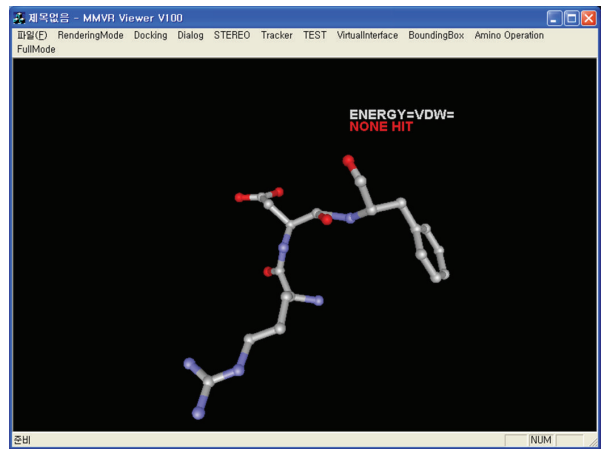

(d)

FIGURE 4: Results of editing operations: (a) an original model on Co-Coot, (b) a changed model on Co-Coot, (c) the original model on VRMMS, and (d) the changed model on VRMMS.

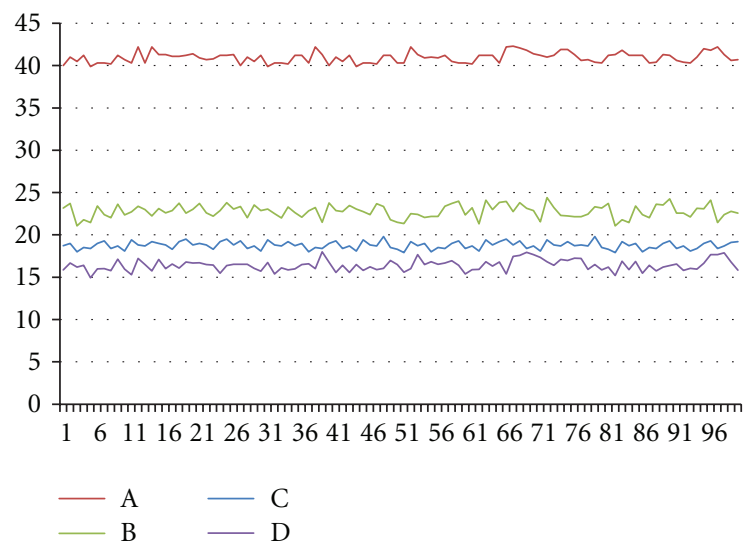

FIGURE 5: Results of various visualization tests: (A) wire frame mode in VRMMS (40.9 fps), (B) ball and stick mode in VRMMS (22.8fps), (C) wire frame mode in Co-Coot (18.8 fps), and (D) surface mode in VRMMS (16.4 fps).

rendering speed even though it is dependent on visualization modes or modeling systems.

4.2. Collaborative Tunneling Test. Second, we measured the number of transformations and average delivering times. In order to measure the values, we conducted a collaborative molecular design process within different environments through network connections as shown in Figure 6. The design computers were placed in different locations, and connected by a $10 \mathrm{Mbps}$ LAN. We selected two experts who have experiences of using VRMMS and Co-coot. They conducted the collaborative design tasks as described in Tables 1 and 2 for 40 minutes.

Figure 6 shows the results of the experiments for the proposed collaborative environments. A total number of transformed operations was 1004, and most occurred transformations were manipulation and chatting as shown in Figure 7(a). As shown in Figure 7(b), the proposed transformation strategies as described in Table 3 showed different delivering performances. Manipulation, ownership, file transferring, and chatting showed fast delivery times. Since Modification needs more times to convert different data, the average delivering time of rendering showed medium performances. Simulation and editing generally showed the worst performances in the experiment because the proposed environment consumed lots of times to transform the operations.

4.3. User Interviews for Acceptance Test. We conducted user interviews with the two subjects after conducting the previous experiments. The participants answered that the proposed environment was generally satisfied. However, they required more collaborative tunneling services such as a voice chatting and a sharing movie. They also answered if the proposed environment could be applied to support 


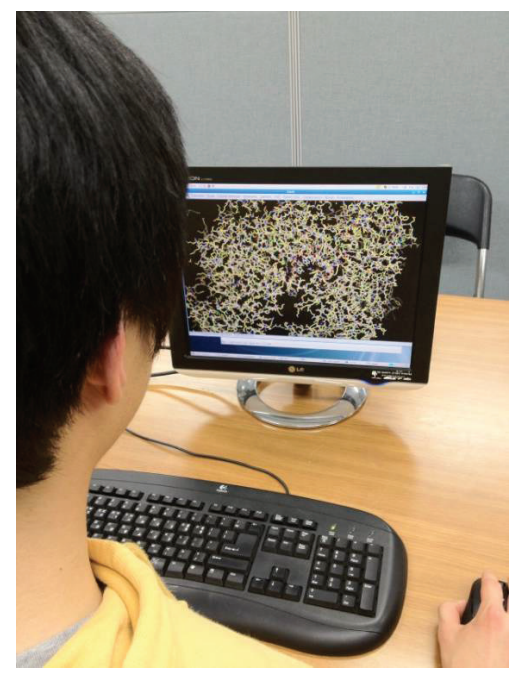

(a)

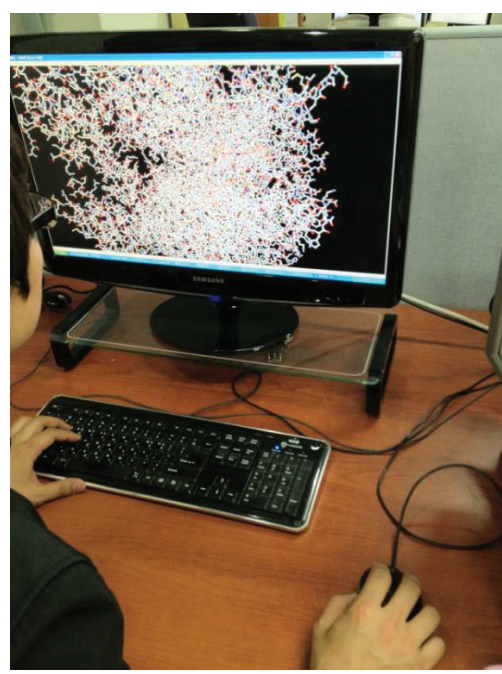

(b)

FIgURE 6: Collaborative molecular modeling: (a) a user is using Co-Coot; (b) another user is using VRMMS.

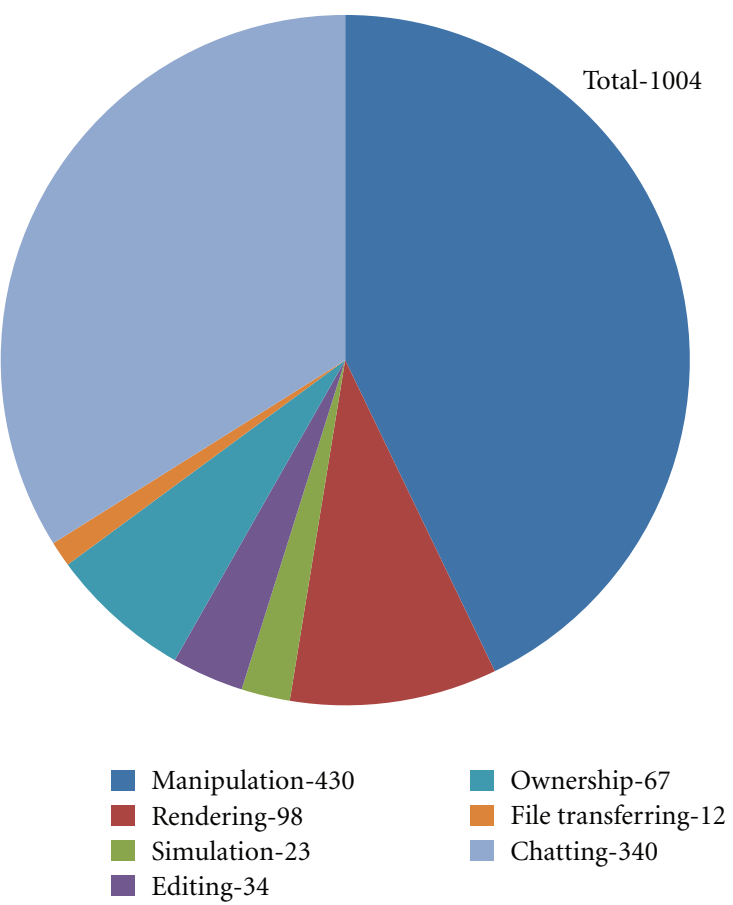

(a)

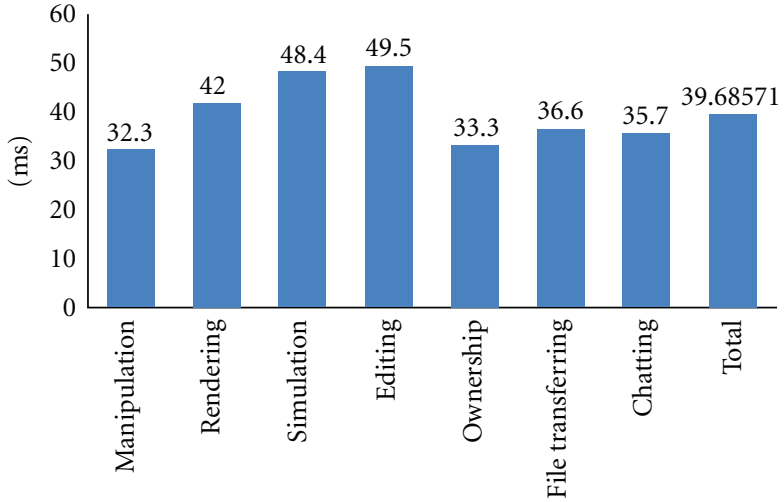

(b)

FIGURE 7: Results of trcollaborative tunneling test: (a) numbers of transformed data according to transformer strategies; (b) average delivering times.

other famous molecular modeling systems, then it would be feasible to utilize the proposed environment in the real collaborative molecular modeling processes.

\section{Conclusions and Future Works}

In this paper, we propose a new collaborative molecular modeling environment to connect different modeling systems based on an approach with a collaborative tunneling service and transformation strategies. With our approach, multiple users can collaborative together even though they are manipulating different modeling systems.

The proposed environment showed feasible rendering performances in target applications with various visualizations. The results of another performance evaluation of the pilot tests showed that the proposed environment could successfully transform collaboration data with stable delivering times through network. 
An additional user study showed that the participants would like to adopt the proposed system in their collaborative molecular modeling environment. For our future works, we will expand the tunneling services to the other popular molecular modeling systems using ontology to support semantic transformation strategies. We also plan to enhance a concurrency control mechanisms with different roles and applications.

\section{Acknowledgment}

This paper was supported by Konkuk University in 2011.

\section{References}

[1] C. Joslin, T. Di Giacomo, and N. Magnenat-Thalmann, "Collaborative virtual environments: from birth to standardization," IEEE Communications Magazine, vol. 42, no. 4, pp. 2833, 2004.

[2] H. Y. Kan, V. G. Duffy, and C. J. Su, "An Internet virtual reality collaborative environment for effective product design," Computers in Industry, vol. 45, no. 2, pp. 197-213, 2001.

[3] A. R. Leach, Molecular Modelling: Principles and Applications, 2001.

[4] K. I. Ramachandran, G. Deepa, and K. Namboori, Computational Chemistry and Molecular Modeling: Principles and Applications, 2008.

[5] J. I. Kim, S. Park, J. Lee, Y. Choi, and S. Jung, "Development of a gesture-based molecular visualization tool based on virtual reality for molecular docking," Bulletin of the Korean Chemical Society, vol. 25, no. 10, pp. 1571-1574, 2004.

[6] S. Park, J. Lee, and J. I. Kim, "A collaborative virtual reality environment for molecular modeling," Advances in Artificial Reality and Tele-Existence, vol. 4282, pp. 324-333, 2006.

[7] J. Lee, P. Quy, J. I. Kim, L. W. Kang, A. Seo, and H. Kim, "A collaborative virtual reality environment for molecular biology," in Proceedings of the International Symposium on Ubiquitous Virtual Reality (ISUVR '09), pp. 68-71, July 2009.

[8] J. Lee, L. W. Kang, H. Kim, and J. I. Kim, "Co-Coot: a real-time collaborative tool for bio-molecular modeling and visualization," in Proceedings of the IEEE International Symposium on Virtual Reality Innovations (ISVRI '110, pp. 281-286, March 2011.

[9] J. Bhandarkar, G. Budescu, W. F. Humphrey et al., "BioCoRE: a collaboratory for structural biology," in Proceedings of the SCS International Conference on Web-Based Modeling and Simulation, pp. 242-251, 1999.

[10] W. Humphrey, A. Dalke, and K. Schulten, "VMD: visual molecular dynamics," Journal of Molecular Graphics, vol. 14, no. 1, pp. 33-38, 1996.

[11] L. V. Kale, M. Bhandarkar, R. Brunner, N. Krawetz, J. Phillips, and A. Shinozaki, "NAMD: a case study in multilingual parallel programming," in Proceedings of the 10th International Workshop on Languages and Compilers for Parallel Computing, pp. 367-381, 1999.

[12] P. Emsley and K. Cowtan, "Coot: model-building tools for molecular graphics," Acta Crystallographica Section D, vol. 60, no. 12, pp. 2126-2132, 2004.

[13] Open API, http://en.wikipedia.org/wiki/Open_API.

[14] K. D. Westover, D. A. Bushnell, and R. D. Kornberg, "Structural basis of transcription: separation of RNA from DNA by RNA polymerase II," Science, vol. 303, no. 5660, pp. 1014 1016, 2004. 

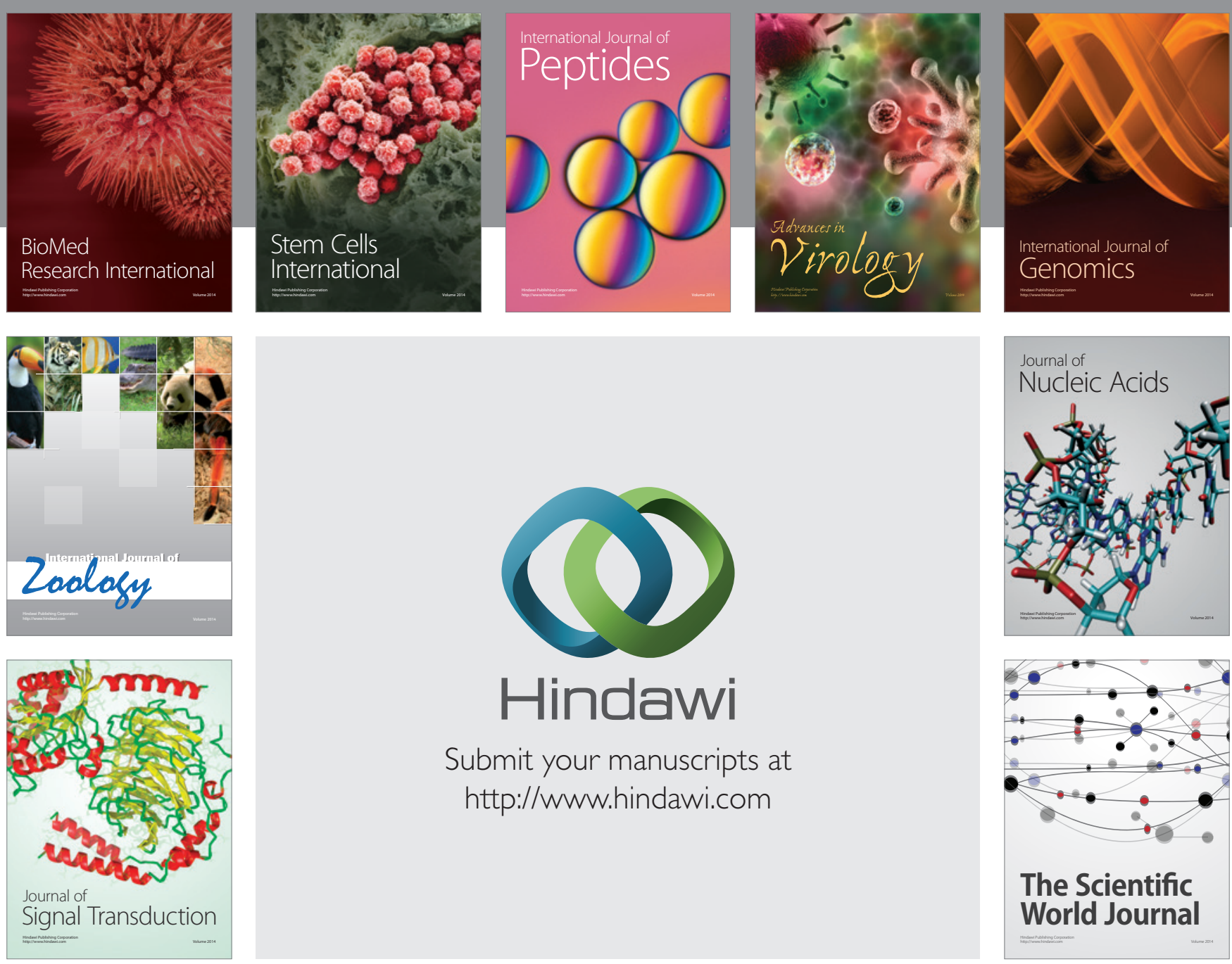

Submit your manuscripts at

http://www.hindawi.com
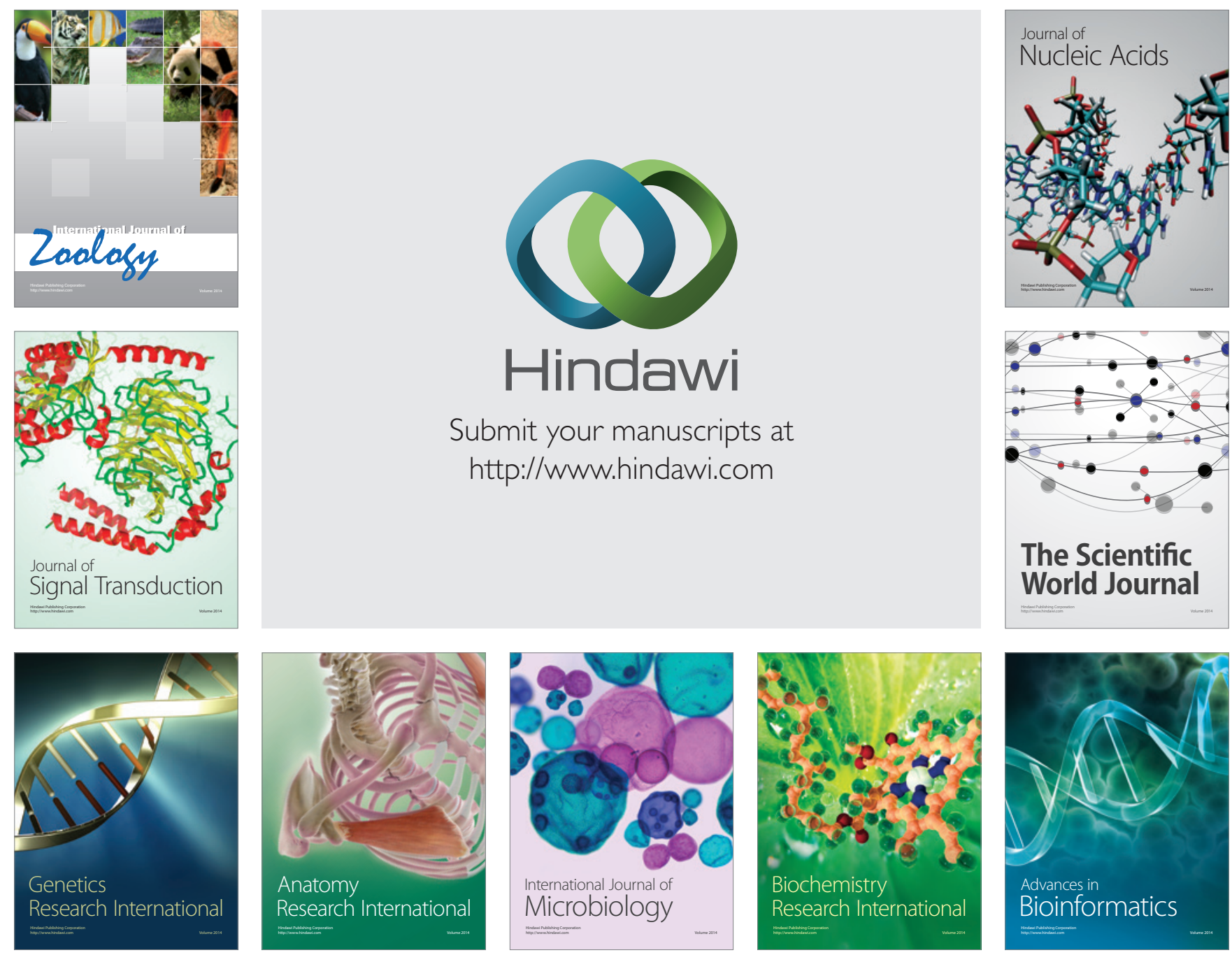

The Scientific World Journal
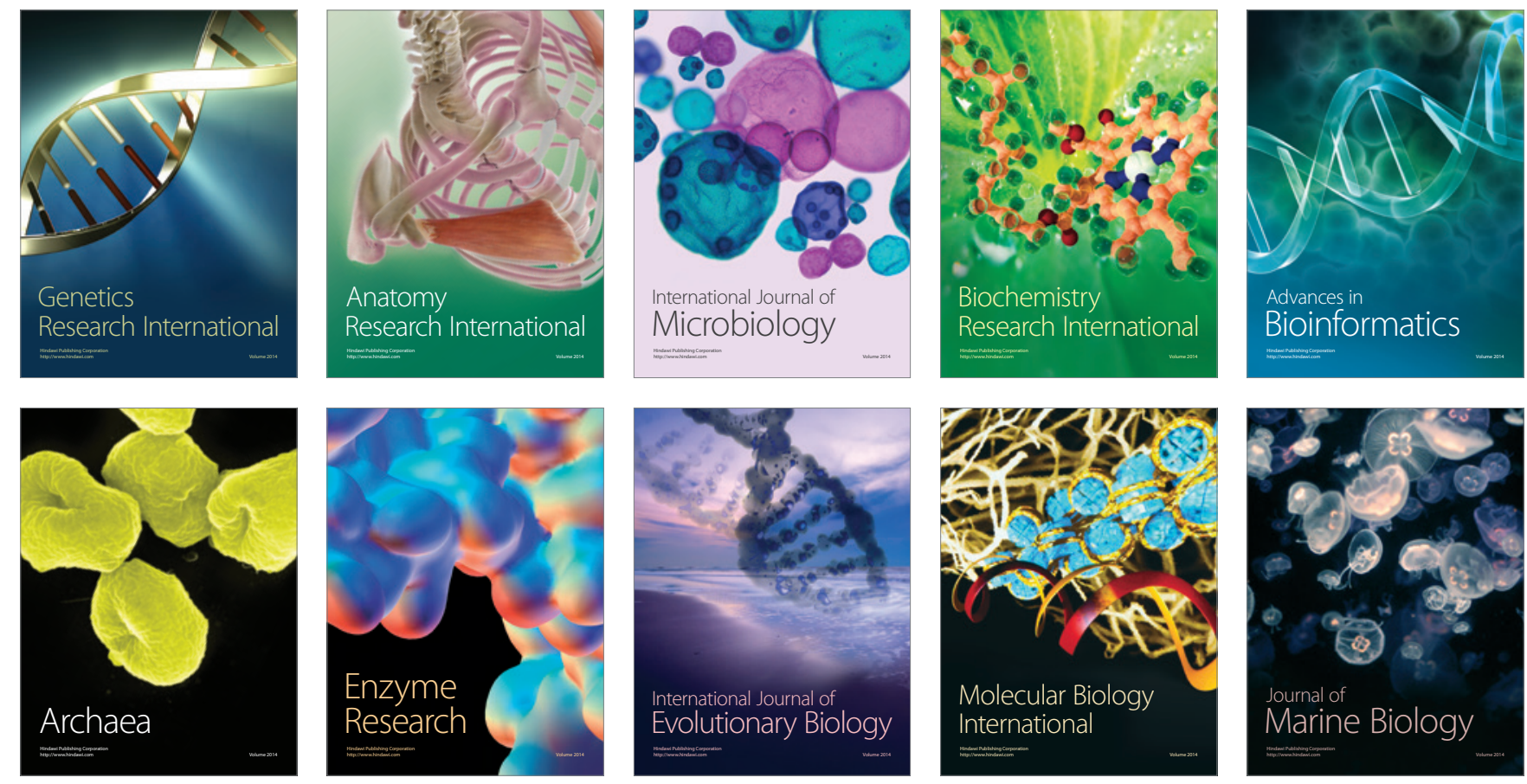